\title{
It's a (stylized) fact!
}

What is a 'stylized' fact? I expect that for most people, including most physicists, this is a rather mysterious term. Is it a fact with style, or according to a certain fashion? Aren't facts just facts? Are some facts more equal than others? To the last question, most of us would say 'yes' - we're most interested in important facts. But this is where things get tricky. What counts as important?

'Stylized facts' is a term well known to physicists in the field of econophysics the application of methods of physics to problems in economics and finance where this peculiar label does, in effect, mean 'important fact'. But important in a way that economists have strangely found uninteresting.

Economist Nicholas Kaldor originally introduced the term 'stylized facts' in 1961, when arguing about theories of economic growth. He made the sensible argument that scientists building a theory should begin from a summary of the relevant facts requiring explanation. Facts first, theory second. But "facts as recorded by statisticians," Kaldor noted, "are always subject to numerous snags and qualifications, and for that reason are incapable of being summarized". Hence, he suggested, theorists would do well to work from "a stylized view of the facts". They should "concentrate on broad tendencies, ignoring individual detail".

Broad tendencies are very much facts deserving attention, and establishing such facts in finance and economics has been a primary achievement of econophysics. Many such facts have been established with appreciable precision only in the past 5-10 years, and they make up a set of more or less established truths that any putative theory of markets ought to explain.

At the outset, consider the basic statistics of market fluctuations. Market movements are of course highly unpredictable in their direction, yet the statistics of how prices change over a certain time interval - say, a few minutes or a single day - turn out to be profoundly regular. The first studies of the distribution of such changes were done in the 1960s by Benoit Mandelbrot with only a few thousand data points. In the 1990s, physicists used more than 200 million data points spanning half a century to establish a strong case for an inverse fourth-power law of stock-market returns, at least asymptotically for large values (corresponding to big market movements).

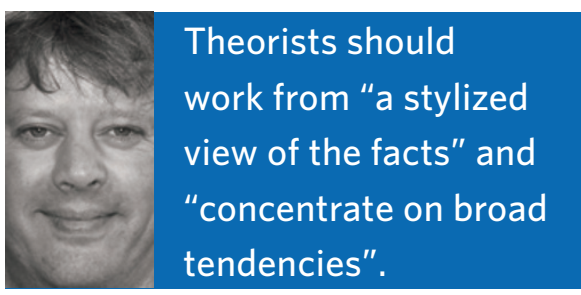

That is, if $R(\Delta t)$ is the return over an interval $\Delta t$ - essentially, the fractional change in the stock price - then the probability distribution of $R$ has a power-law tail of the form $P(R) \sim 1 / R^{4}$. The simplicity of this form is in itself quite surprising. Perhaps even less expected is that the same form holds for intervals varying from a second up to several days, in different kinds of markets - for stocks, foreign exchange, futures and so on - as well as in markets in many different countries.

This pattern is akin to the Maxwellian distribution of velocities in a gas - a general law holding for all markets, although we don't at present know why. It implies, among other things, that markets are especially prone to large, sudden fluctuations. A change of $20 \%$ in the price of a stock is a move of about ten standard deviations, as individual stocks typically change about $2 \%$ in a day. Market data show that moves this big happen essentially every week for at least one of the few thousand stocks in the market (X. Gabaix, Annu. Rev. Econ. 1, 255-293; 2009). It even appears that the crashes of 1929 and 1987 fit quite naturally within this distribution, suggesting that there may be no need for any special explanation for such crashes - they may be just big but otherwise normal stock movements.

Now, the exponent in this pattern isn't always found to be exactly four, but varies slightly from one market to another. This isn't quite the accuracy of the inverse-square law for gravity, but for a phenomenon in the social sciences, this is still impressive. It's a fact, not merely a 'stylized' fact.

And it is one of many similar facts. Some abrupt market movements seem to be strongly linked to external news, such as an earthquake or oil discovery. Others don't seem to be so directly caused by news, but find their origin in the big trade of some market participant, done for reasons known only to them. As it turns out, markets behave very differently in the aftermath of these two kinds of events, yet in each case have a very simple behaviour. Plot the excess market volatility (over the long-run average) versus time after a big event caused by some obvious news, and you find that the deviation decays in proportion to $1 / t$. Do the same thing just after a big event not caused by news, and you instead find decay in proportion to $1 / t^{1 / 2}$. Of course, the latter form implies a much slower relaxation back to the norm.

Hence, it seems that the market takes a longer time to get back to normal after a no-news jump, whereas it goes back to normal quite quickly after a news-related jump (A. Joulin et al., http://arXiv.org/ abs/0803.1769; 2008). No one knows why this should be, but one proposal is that a market jump clearly related to news is not really surprising. Traders and investors soon get on with their usual business. In contrast, a no-news event is very different. It is a real shock and presents a lingering unexplained mystery. It is unnerving and makes investors uneasy. The resulting uncertainty registers in lingering high volatility.

These two 'stylized' facts - one on the distribution of returns, the other on a universal pattern of market relaxation only scratch the surface of a vast range of mathematical patterns suggesting some deep order within some of our most chaotic social systems. Another 'stylized' fact is a regularity in the day-to-day fluctuations in market volatility. Studies show that episodes of high volatility work very much like earthquakes, following the same mathematical pattern the Omori law - that seismologists use to determine the likelihood of an aftershock in the days and weeks following a main shock. As with earthquakes, periods of high and low volatility cluster together, in a precise mathematical relationship.

The distribution of firms by size follows another power law (very similar in different nations), as does the distribution of firm lifetimes (very similar also to the distribution of species lifetimes in the fossil record). All these facts demand explanation by any decent market theory. Indeed, theories worth exploring should generate these regularities as a matter of course, much as physical theories should conserve energy and basic symmetries.

Oddly, they have until very recently been little explored by economists, and considered of lesser importance because they are only 'stylized'.

MARK BUCHANAN 